\title{
Surface-Modified Bacterial Cellulose with Mercaptosilane as a Multifunctional Platform
}

\author{
Nayara C. do Amaral ${ }^{1 *}$, Amanda M. Claro ${ }^{1 *}$, Gustavo C. Monteiro², Hernane S. Barud ${ }^{1}$ \\ ${ }^{*}$ Corresponding author: E-mail address: nayaraamaral1234@gmail.com
}

Abstract: Cellulose synthesized by bacteria has unique properties such as high water retention capacity, biocompatibility, biodegradability and flexibility. Nevertheless, modification of this biomaterial is required in order to obtain multifunctional materials, which may be applied in several high-value added products, as catalytic and cell culture platforms. The surface of bacterial cellulose (BC) can be modified by several approaches, namely: (i) physical treatment by plasma, (ii) adsorption of molecules onto BC surface, and (iii) chemical modification. In this sense, the aim of this study was to modify the BC surface by silanization reaction at room temperature using a mixture of ethanol and water, using two different protocols. Thus, BC membranes synthesized by Komagataeibacter xylinus were modified by adding the thiol (SH) functional group with (3-mercaptopropyl) trimethoxysilane under mild conditions. The produced materials were analyzed by elemental analysis, ATR-FTIR, TGA and SEM, and the successful modification was proven by elemental analysis and SEM.

Keywords: Bacterial cellulose; Surface Modification; Silanization; Mercaptosilane.

\section{Introduction}

Cellulose is the most abundant biopolymer on planet and it can be produced by green plants, fungi and bacteria. Cellulose consists of $\beta$-D-glycopyranose units linearly arranged by $\beta-(1-4)$ glycosidic bonds. Plant-derived cellulose is usually associated with several components as hemicellulose, lignin and pectin, while bacterial cellulose (BC) is obtained chemically pure. Besides, BC presents nanometer-sized fibers once it is excreted by a microorganism, therefore $\mathrm{BC}$ is also known as bacterial nanocellulose ${ }^{(1)}$. BC has several unique properties such as biocompatibility, mechanical strength, biodegradability, high water retention capacity and flexibility ${ }^{(2)}$, hence $\mathrm{BC}$ is a promising material whenever these properties are required.

Because of its distinct properties, $\mathrm{BC}$ has gained prominence in the scientific field, especially in the biomedical area as wound dressings, matrix for controlled drug release and temporary skin substitute ${ }^{(2)}$. However, the introduction of either charged or hydrophobic moieties onto the bacterial nanocellulose is necessary in many applications ${ }^{(3-4)}$. Ideally, the modifications should be effective without altering the biomaterial nanostructure, once it accounts for the material distinct properties.

Chen et al. ${ }^{(3)}$ have functionalized the BC surface with amidoxime; the researchers have produced a nanohybrid based on the amidoxime surface functionalized BC and gold nanoparticles, which had shown excellent catalytic activity. Taokaew et al. ${ }^{(4)}$ have chemically modified the $\mathrm{BC}$ surface by grafting methyl and amine terminated organosilanes to evaluate attachment, spreading and growth of normal human dermal fibroblasts; it was observed that the cells improved their spreading and attachment on the amine-modified BC. Li et al. (5) have introduced the thiol group into cellulose chains, creating a platform capable of undergo further modifications via thiol-ene reaction.

Traditionally, surface modification protocols to impart hydrophobic properties ${ }^{(6)}$ onto nanocellulose do not respect the green-chemistry principles. Besides being time-consuming, most of them use to employ previous solvent-exchange steps with hazardous aprotic organic solvents such as dimethyl sulfoxide and $N, N$-dimethylformamide in addition to heat curing process after the chemical treatment ${ }^{(7)}$.

This study aims to evaluate the efficiency of BC surface modification through silanization reaction under room temperature and using a mixture of ethanol and water as solvent through two simple protocols which do not require specific apparatus. Particularly, two different silanization methods were employed in order to modify the BC surface by adding the thiol (SH) functional group, an active site for further modification, with (3-mercaptopropyl)trimethoxysilane (MPTMS): Method A, according to the protocol described by Frone et al. ${ }^{(8)}$, and Method $\mathrm{B}$ using $\mathrm{NH}_{4} \mathrm{OH}$, as described by Lu et al. ${ }^{(9)}$.

\section{Experimental Procedures}

BC Production and Surface Modification

The strain used for the BC production was Komagataeibacter xylinus. The bacteria were cultured under static conditions in HestrinSchramm (HS) media, composed of D-glucose, yeast extract, peptone, disodium hydrogen phosphate, citric acid, and distilled water.

Dried BC membranes of surface area and thickness of $1.5 \mathrm{~cm}^{2}$ and $0.05 \mathrm{~mm}$, respectively, were treated by silanization reaction with MPTMS by two different methods. A mixture of ethanol and water 10:1 (v/v) was used as solvent.

For Method A (sample BC-SH-A), it was used $0.538 \mathrm{mmolmL}^{-1}$ of MPTMS. The system was kept at stirring for four hours at room temperature. Then the excess of solution was discarded and the membranes were washed three times with acetone and dried at room temperature for three days ${ }^{(8)}$.

For Method B (sample BC-SH-B), the membranes were put in contact with the solvent for 5 minutes. Then $\mathrm{NH}_{4} \mathrm{OH}$ was added to medium followed by the addition of siloxane - the final medium concentration was $0.163 \mathrm{mmolmL}^{-1}$ of MPTMS, three times lower than that used in the first method. The system was kept at stirring for 12 hours at room temperature. Then, as the process done for the previous method, the excess of solution was discarded and the membranes were washed three with acetone and dried at room temperature for three days ${ }^{(9)}$.

\footnotetext{
1 University of Araraquara - UNIARA, Araraquara, SP, Brazil.

2 TechMiP Análises e Soluções Inteligentes LTDA.
} 


\section{Characterization}

The membranes were characterized by elemental analysis, Attenuated Total Reflectance Fourier Transform Infrared (ATR-FTIR), Scanning Electron Microscopy (SEM) and Thermogravimetric Analysis (TGA).

\section{Elemental Analysis}

Hydrogen, sulfur, nitrogen and carbon contents were determined by dry combustion in a Perkin Elmer elemental analyzer, model 2400 series Il.

\section{ATR-FTIR}

The transmittance spectra were obtained in the infrared region by attenuated total reflectance (ATR-IR) in a Cary 630 Agilent spectrometer in the range of $4,000-650 \mathrm{~cm}^{-1}$.

\section{SEM}

The morphological characterization was performed using a Joel JSM 7500F Field Emission Scanning Electron Microscope. The samples were covered with a thin layer of conductive carbon. The images were taken at 10,000 times magnification.

\section{TGA}

Thermogravimetric analyzes were performed on a TA Instruments SDT Q600 thermal analyzer under the following conditions: heating rate of $10{ }^{\circ} \mathrm{C} / \mathrm{min}$ with a synthetic air flow of $100 \mathrm{~mL} / \mathrm{min}$, from $30^{\circ} \mathrm{C}$ to 600 ${ }^{\circ} \mathrm{C}$. Alumina pan was used as reference.

\section{Results and Discussion}

In terms of chemical reaction of silanization, water has the function of induce siloxane hydrolysis to silanol (Figure 1, Step 1), while ethanol favors the materials drying process. The interaction between hydroxyls favors the condensation reaction (Figure 1, Step 2). Thus, in this type of modification the condensation reaction occurs between silanol groups
$(\mathrm{Si}-\mathrm{OH})$ as well as between the silanol and the $\mathrm{BC}$ hydroxyls ${ }^{(10)}$. The condensation step can also be induced by employing strong or weak bases such as $\mathrm{NaOH}^{(7)}$ and $\mathrm{NH}_{4} \mathrm{OH}^{(9)}$.

The efficiency of $\mathrm{BC}$ surface modification by the applied methods was verified by elemental analysis of pristine $\mathrm{BC}$, used as reference, compared to the treated samples (Table 1).

Modifying the material with MPTMS necessarily implies increasing sulfur (S) content once this siloxane carries the thiol functional group. $\mathrm{BC}-\mathrm{SH}-\mathrm{A}$ has a sulfur percentage very close to the native (reference) while $\mathrm{BC}-\mathrm{SH}-\mathrm{B}$ has a sulfur percentage almost 4 times higher compared to the native. This indicates that only method $B$ was indeed efficient in modifying the BC surface through the covalent $\mathrm{Si}-\mathrm{O}-\mathrm{Si}$ bond formed between the silane moiety and the polymeric matrix.

FTIR-ATR spectrum (Figure 2) of native BC and BC treated by Methods $A$ and $B$ have the same profile. In all of them, there is a weak band at $900 \mathrm{~cm}^{-1}$, attributed to vibrational modes of the $\mathrm{C}-0-\mathrm{C}_{\beta}(1 \rightarrow 4)$ glycosidic bonds between glucose units, a band at $1,054 \mathrm{~cm}^{-1}$ attributed to the $\mathrm{C}-\mathrm{OH}$ stretch, and a band at 1,163 $\mathrm{cm}^{-1}$, attributed to asymmetric deformation of the glucose pyranose ring $\mathrm{C}-0-\mathrm{C}$. The $3,340 \mathrm{~cm}^{-1}$ band is attributed to the $\mathrm{O}-\mathrm{H}$ stretch of water adsorbed to the cellulose surface ${ }^{(11)}$. This analysis was not sensitive attest the surface functionalization even for the BC treated by Method B, once it was not possible to observe the band corresponding to the deformation of the $\mathrm{Si}-0$-Cellulose group in the region between $1,100-1200 \mathrm{~cm}^{-1(8)}$.

In the SEM images obtained from the sample surfaces (Figure 3), it is observed that the native $\mathrm{BC}$ has a three-dimensional nanometric structure with randomly arranged nanofibers. It can be seen that the surface of the sample submitted to Method A did not change (there was no modification), unlike the surface of the sample submitted to Method $\mathrm{B}$, where a distinct structure material deposited on the nanofibers is observed. These results corroborate with the data obtained by elemental analysis.

According to the thermogravimetric curves (Figure 4), it can be seen<smiles>CO[Si](CCCS)(OC)OC</smiles>
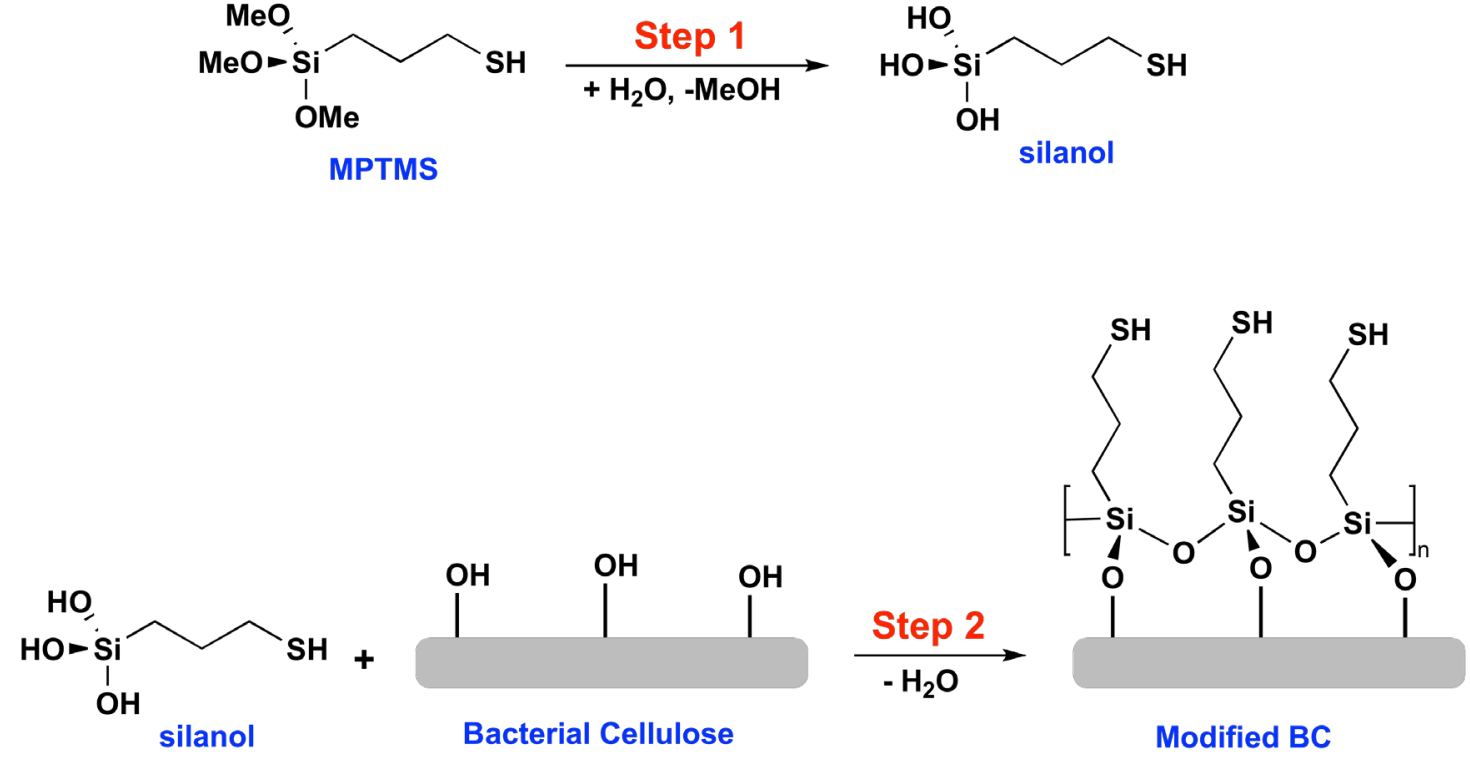

Figure 1 - Silanization reaction with MPTMS. 
Amaral et al.

\begin{tabular}{c|c|c|c|c}
\hline Sample & Hydrogen (\%) & Nitrogen (\%) & Sulfur (\%) & Carbon (\%) \\
\hline Native BC & 5.75 & 0.87 & 1.04 & 42.45 \\
\hline BC-SH-A & 5.95 & 0.94 & 1.37 & 42.23 \\
\hline BC-SH-B & 6.26 & 0.91 & 3.3 & 40.16 \\
\hline
\end{tabular}

Table 1 - Elemental composition of samples.

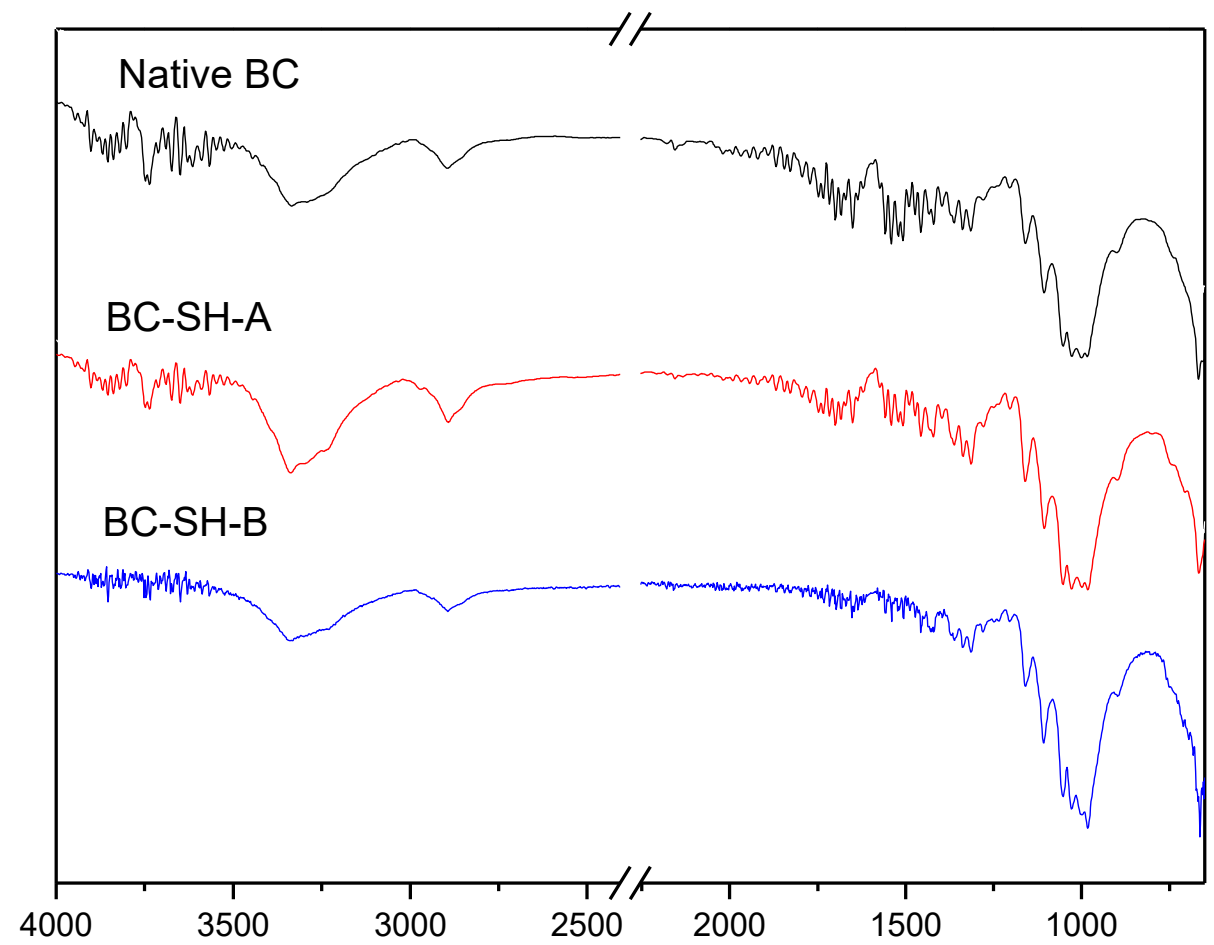

Figure 2 - ATR-FTIR spectrum of Native BC, BC-SH-A, and BC-SH-B. 
Surface-Modified Bacterial Cellulose...

(A)

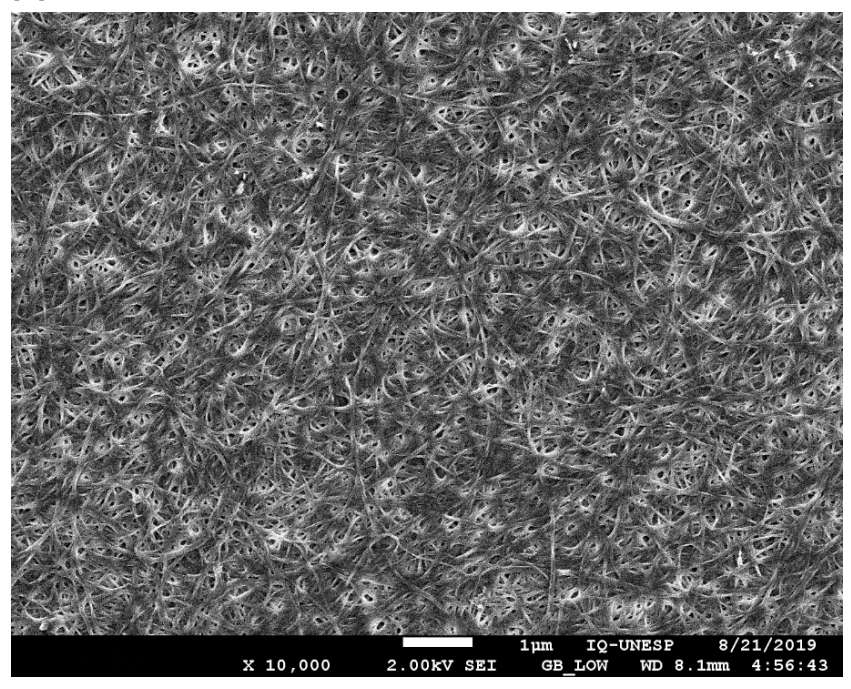

(B)

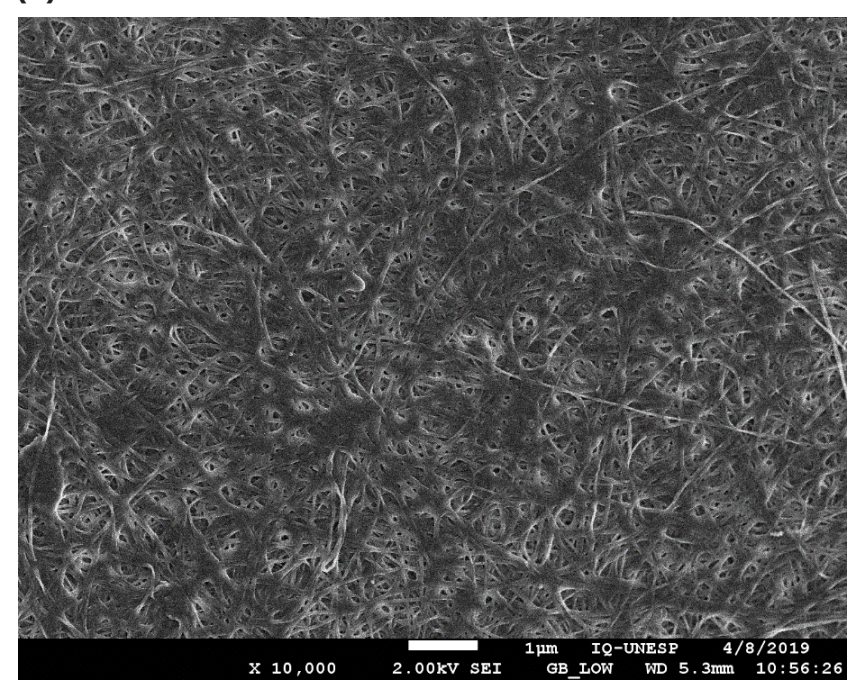

(C)

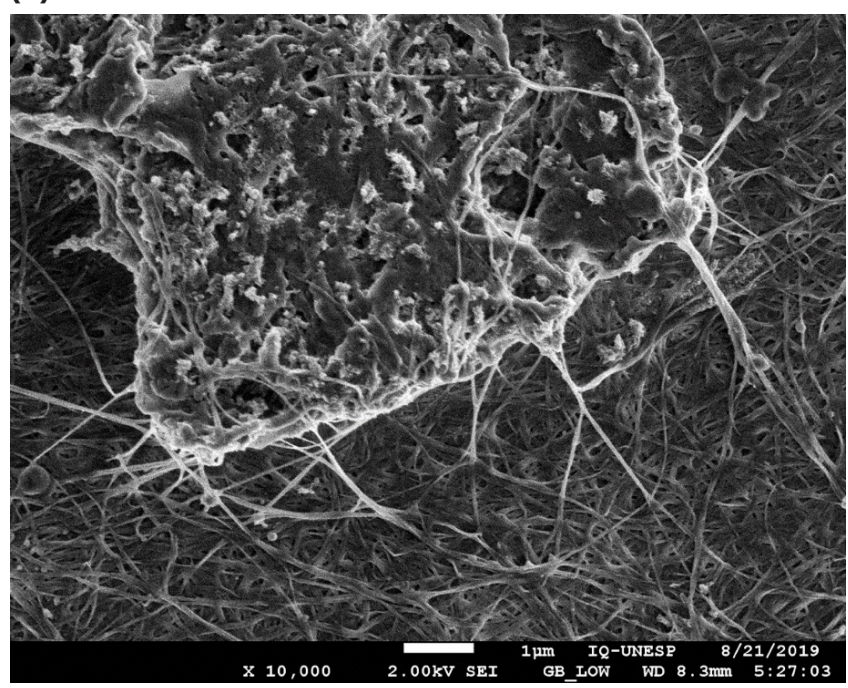

Figure 3 - Surface image by SEM of (A) Native BC (B) BC-SH-A and (C) BC-SH-B. 
that the membranes treated by both Methods show mass losses with more pronounced inflection points in relation to native BC. In addition, the percentage of surface water loss is lower for treated membranes: about $3 \%$ for treated samples and $5 \%$ for BC. As well as the FTIR analysis, the TGA was not sensitive to verify the $B C$ functionalization once it was not observed mass increase by $600^{\circ} \mathrm{C}$ due to silicon oxide formation.

\section{(A)}

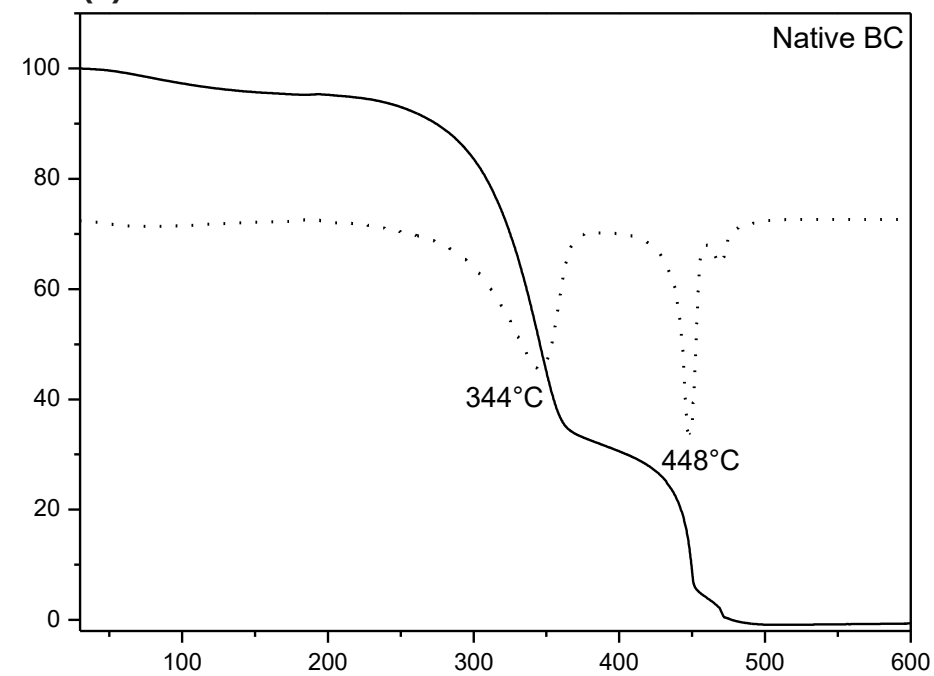

(B)

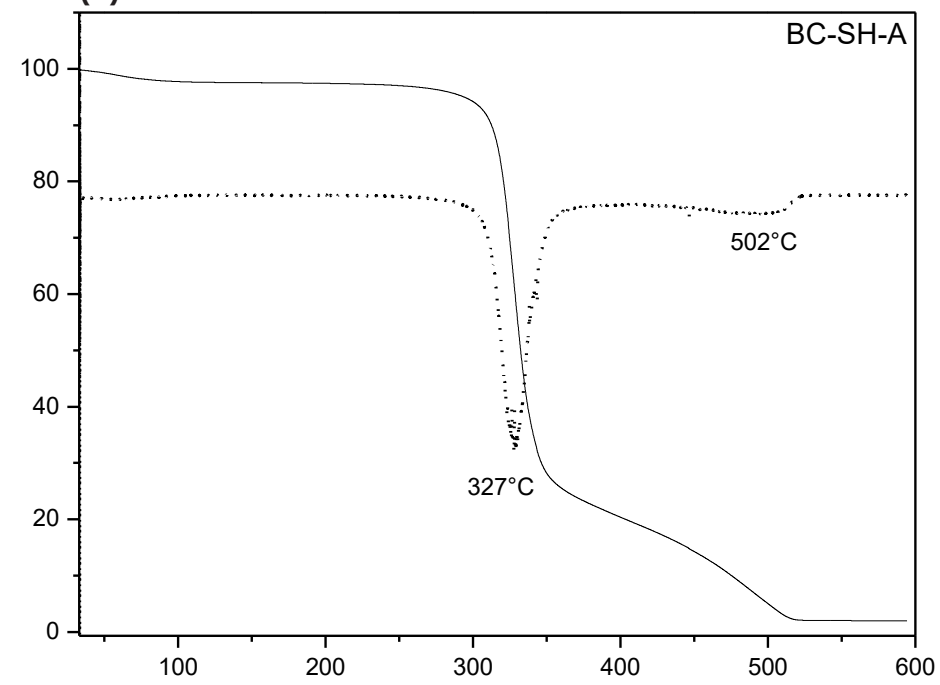

(C)

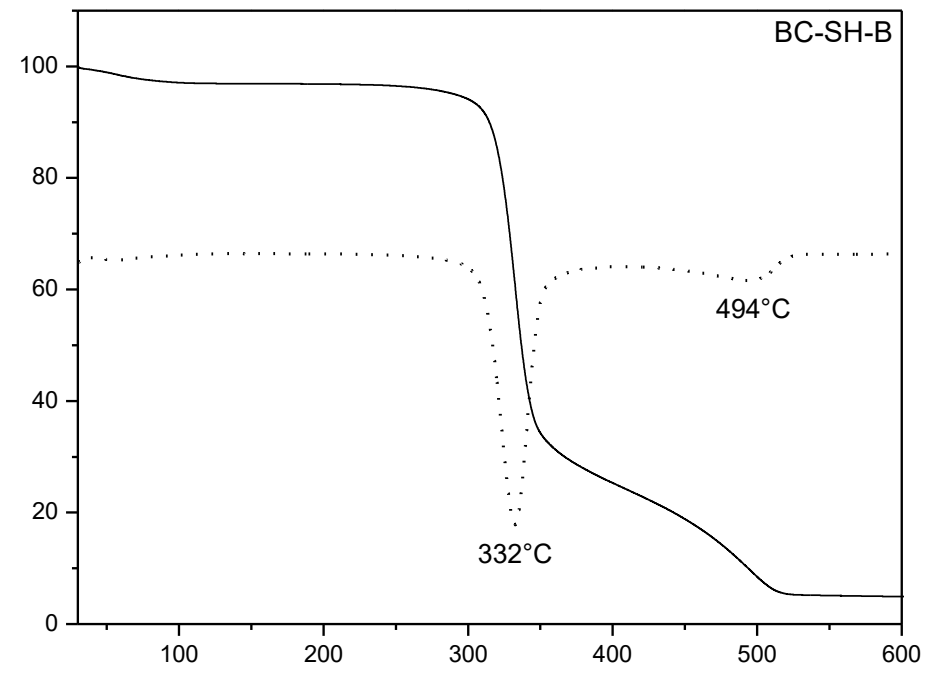

Figure 4 - TGA and DTG curves of (A) Native BC (B) BC-SH-A and (C) BC-SH-B. 


\section{Conclusions}

Among the silanization methods employed in this study for BC surface modification, only the one whose condensation step was induced by employing $\mathrm{NH}_{4} \mathrm{OH}$ was indeed effective. Elemental analysis and SEM analysis confirmed the efficiency of this particular surface functionalization using MPTMS.

\section{Funding and Acknowledgments}

The authors gratefully acknowledge TA Instruments Brazil and the financial support of São Paulo Research Foundation (FAPESP, Process\#2018/23853-2 and \#2019/12711-5), National Institute of Photonics (INFo) and Brazilian Higher Education Improvement Coordination (CAPES)

\section{References}

1. Donini ÍAN, De Salvi DTB, Fukumoto FK, Lustri WR, Barud HS, Marchetto R, Messaddeq Y, Ribeiro SJL. Biosynthesis and recent advances in bacterial cellulose production. Eclet. Quím. 35 :165-178 (2010). DOI: 10.1590/S0100-46702010000400021.

2. Trovatti E, Dos Santos AM, Amaral AC, Meneguin AB, Matos BDM, Pacheco G, et al. Biopolymers: pharmaceutical and biomedical applications. Eclet. Quím. 41 :1-31 (2016). DOI: 10.26850/16784618eqj.v41.1.2016.p01-31.

3. Chen M, Kang H, Gong Y, Guo J, Zhang H, Liu R. Bacterial cellulose supported gold nanoparticles with excellent catalytic properties. ACS Applied Materials \& Interfaces. 7 :21717-21726 (2015). DOI: 10.1021/acsami.5b07150.

4. Taokaew S, Phisalaphong M, Newby BZ. Modification of bacterial cellulose with organosilanes to improve attachment and spreading of human fibroblasts. Cellulose. 22 :2311-2324 (2015). DOI: $10.1007 / s 10570-015-0651-x$

5. Li W, Wang W, Yang Y, Zhang K. Redox-responsive, reversibly fluorescent nanoparticles from sustainable cellulose derivatives. J. Mater. Chem. A. 2 :13675-13681 (2014). DOI: 10.1039/c4ta02126f.

6. Missoum K, Belgacem MN, Barnes JP, Brochier-Salon MC, Bras J. Nanofibrillated cellulose surface grafting in ionic liquid. Soft Matter 8 :8338-8340 (2012). DOI: 10.1039/C2SM25691F.

7. Beaumont M, Bacher M, Opietnik M, Gindl-Altmutter W, Potthast A, Rosenau T. A General Aqueous Silanization Protocol to Introduce Vinyl, Mercapto or Azido Functionalities onto Cellulose Fibers and Nanocelluloses. Molecules 23 :1-15 (2018). DOI: 10.3390/molecules23061427.

8. Frone AN, Panaitescu DM, Chiulan I, Nicolae CA, Casarica A, Gabor AR, et al. Surface treatment of bacterial cellulose in mild, eco-friendly conditions. Coatings 8 :3-17 (2018). DOI: 10.3390/ coatings 8060221

9. Lu F, Li H, Sun M, Fan L, Qiu H, Li X, Luo C. Flow injection chemiluminescence sensor based on core-shell magnetic molecularly imprinted nanoparticles for determination of sulfadiazine. Anal. Chim. Acta. 718 :84-91 (2012). DOI: 10.1016/j.aca.2011.12.054.

10. Salon MCB, Abdelmouleh M, Boufi S, Belgacem MN, Gandini A. Silane adsorption onto cellulose fibers: Hydrolysis and condensation reactions. J. Colloid Interface Sci. 289 :249-261 (2005). DOl: 10.1016/j.jcis.2005.03.070.

11. De Salvi DTB, Barud HS, Caiut JMA, Messaddeq Y, Ribeiro SJL.
Self-supported bacterial cellulose/boehmite organic-inorganic hybrid films. J Sol-Gel Sci Technol. 63 :214-2015 (2012). DOI:10.1007/s10971-012-2678-x. 